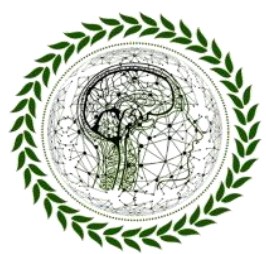

PhD Scientific Review

Artigo submetido em: 15/06/2021

ISSN 2676 - 0444

Artigo aceito em: 18/06/2021

Artigo publicado em: 19/06/2021

\title{
SUICÍDIO DE IDOSOS: PREVENÇÃO E CUIDADO DA SAÚDE MENTAL NA FASE DO ENVELHECIMENTO
}

\author{
Hermanna Maria Silva Nunes, UNIFSA ${ }^{1}$ \\ Naimara Keze Nunes Oliveira, UNIFSA ${ }^{2}$ \\ Karinne De Araújo Paiva, UNIFSA ${ }^{3}$ \\ Patrícia Melo do Monte,UNIFSA ${ }^{4}$
}

\section{RESUMO}

O suicídio ainda é considerado tabu na sociedade brasileira. Esse fenômeno tem se elevado entre os idosos no Brasil; assim, a pesquisa teve como objetivo geral conhecer a produção científica a respeito da temática do suicídio de idosos, identificar fatores de risco associados ao suicídio, descrever a ocorrência de transtornos psiquiátricos associados aos casos e relatar intervenções como o papel do cuidador, o tratamento de possíveis problemas psíquicos, entre outros de caráter preventivo. $\mathrm{O}$ artigo trata-se de uma revisão bibliográfica, na base de dados Google Acadêmico, em artigos publicados no período de 2017 a 2021. Como resultados, foram selecionados cinco estudos, e verificou-se que a depressão associada a outros fatores como sofrimentos físicos, questões existenciais, isolamento social, entre outros, potencializam a consumação do suicídio em idosos. Nos casos descritos, há uma prevalência de depressão em comparação com outros fatores de risco para o suicídio nessa fase da vida.

Palavras-chave: Suicídio. Idosos. Transtornos psiquiátricos. Prevenção

\footnotetext{
ABSTRACT

${ }^{1}$ Pós- Graduada em nível de Especialização, na área da Educação, denominado Atendimento Educacional Especializado da Faculdade do Meio Norte; Licenciada em Pedagogia. Professora da Escola Municipal José Auto de Abreu. Graduanda do curso de Psicologia do Centro Universitário Santo Agostinho, hermanna-nunes@hotmail.com

${ }^{2}$ Graduanda do curso de Psicologia do Centro Universitário Santo Agostinho- UNIFSA, naimarakeze@gmail.com

${ }^{3}$ Graduanda do curso de Psicologia do Centro Universitário Santo Agostinho- UNIFSA, karinnepaiva@hotmail.com

${ }^{4}$ Psicóloga, Doutora em Educação. Professora do Centro de Ciências da Saúde da Universidade Estadual do Piauí. Professora do Curso de Psicologia do Centro Universitário Santo Agostinho. Email: patriciamelo2000@gmail.com
}

PhD Scientific Review(C) ISSN 2676 - 0444 
Suicide is still considered taboo in Brazilian society. This phenomenon has increased among the elderly in Brazil; about this, the research had as a general objective to know the scientific production regarding the theme of suicide among the elderly, to identify risk factors associated with suicide, to describe the occurrence of psychiatric disorders associated with the cases and to report interventions such as the role of the caregiver, the treatment of possible psychic problems, among others of a preventive nature. The article is a literature review, in the Google Scholar database, in articles published from 2017 to 2021. As a result, five studies were selected, and it was found that depression associated with other factors such as physical suffering, existential issues, social isolation, among others, enhance the consummation of suicide in the elderly. In the cases described, there is a prevalence of depression compared to other risk factors for suicide at this stage of life.

Keywords: Suicide. Elderly. Psychiatric disorders. Prevention

\section{INTRODUÇÃO}

A terceira idade é considerada uma fase da vida que se inicia por volta dos 60 anos, onde os idosos sofrem com estigmas que se entrelaçam a esse processo, como, a ideia de finitude (proximidade com a morte), perda de papeis na sociedade com a chegada da aposentadoria, onde a própria sociedade estabelece um tempo determinado para a capacidade produtiva, o qual segundo Santos e Encarnação (1997) nos remete ao fato de que não se trata de um envelhecimento biológico a questão exposta acima e, sim de um envelhecimento social que implica na compreensão do homem velho no seu relacionamento com a modernidade, mais precisamente, com os modos de vida da sociedade atual.

Assim, sabendo-se que a população global segue um processo de envelhecimento ao longo do tempo, o combate aos estereótipos se faz necessário como forma de prevenção do idadismo, que é o preconceito ou discriminação com base na idade; pois a fase da terceira idade deve ser percebida com um olhar 
cuidadoso em virtude do fenômeno que vem acometendo tais sujeitos que é o fenômeno do suicídio entre idosos (PAPALIA E FELDMAN, 2013).

O suicídio é um ato proveniente do próprio sujeito que atenta, comete a ação de dar fim a sua vida. Ao nos reportarmos a história ocidental, o suicídio sempre existiu e na atualidade, o suicídio é considerado um problema de saúde pública. Os dados do Ministério da Saúde, divulgados em 2018, apontam para uma taxa alta de suicídio de idosos com mais de 70 anos, sendo registrada uma taxa média de 8,9 mortes por 100 mil nos últimos seis anos, acima da taxa média nacional que é de 5,5 mortes por 100 mil. Mostra-se um fenômeno crescente e representando um problema de saúde pública, vista que os dados do IBGE (2018) indicam que a população brasileira com 60 anos de idade ou mais cresceu 18,8\% entre 2012 a 2017, o que se faz necessário uma atenção maior a população idosa.

Os estudos sobre o suicídio de idosos têm se mostrado escassos quanto à investigação de campo, pois ainda existe um estigma, é visto como tabu que ainda precisa ser desmistificado "o comportamento suicida ainda é obscurecido por tabus", sendo visto como vergonha (BOTEGA, 2015, p.31). Porém, um estudo de campo "o suicídio de idosos em Teresina: fragmentos de autópsias psicossociais" de Teixeira e Martins (2018) deu um embasamento para a discussão na constatação de quais os fatores de riscos para o suicídio de idosos.

Dessa forma, o suicídio mostra-se como um tema aberto a diferentes perspectivas, por ser complexo, com natureza de dilema, multidimensional, em que "não se pode padronizar ou afirmar com total precisão qual a combinação de fatores de riscos que direcionam o idoso a atentar contra a própria vida" (TEIXEIRA E MARTINS, 2018, P.263); Porém, estudos aprofundados mostram fatores de riscos associados ao sofrimento psíquico o qual o idoso esteja acometido que potencializa o suicídio.

Diante disso, esta pesquisa foi realizada com o objetivo de investigar as contribuições das investigações científicas publicadas sobre a realidade brasileira no período de 2017 a 2021 sobre o suicídio de idosos, buscando identificar fatores de risco associados ao suicídio em idosos; descrever a ocorrência de transtornos 
psiquiátricos associados aos casos e relatar intervenções de caráter preventivo realizados nos casos descritos.

Nessa perspectiva, tal pesquisa é de grande importância para a sociedade, pois evidencia fatores de risco e contribui com conhecimento para medidas de prevenção ao suicídio bem como para a melhoria da qualidade de vida das pessoas idosas, além de um olhar também para as pessoas que cuidam dos idosos.

\section{METODOLOGIA}

Trata-se de uma pesquisa qualitativa, do tipo bibliográfica, de caráter exploratória, por meio de uma revisão sistemática, onde Gil $(2008$, p.50) destaca a importância da pesquisa bibliográfica que é "o fato de permitir ao investigador a cobertura de uma gama de fenômenos muito mais ampla do que aquela que poderia pesquisar diretamente"; então, cuidadosamente, foi realizada uma revisão de literatura das publicações nacionais sobre o suicídio de idosos, levantadas na base de dados Google Acadêmico. O método consistiu em sintetizar as principais contribuições dos artigos analisados em eixos temáticos, conforme os objetivos do estudo. Os estudos selecionados incluíram apenas artigos, publicados em língua portuguesa, no período a partir de 2017.

Foram excluídos dissertações, teses, artigos científicos e comunicações em congressos e os textos que não apresentavam o suicídio de idoso como objeto central da reflexão. Foi realizada uma busca pelas associações das seguintes palavras-chave: suicídio, idoso, transtornos psíquicos, prevenção. Os textos selecionados foram organizados, inicialmente, conforme o ano de publicação, o (os) autor (es), o título e o tipo de publicação. Em seguida, as contribuições dos cinco artigos analisados foram identificadas e classificadas em eixos temáticos, a partir da leitura das publicações. 


\section{RESULTADOS E DISCUSSÃO}

Nesse estudo, foram analisados cinco artigos, os mesmos foram dispostos da seguinte forma, como, tabela 1 preenchida com as informações sobre os trabalhos coletados na base de dados Google Scholar e a tabela 2 com os autores, o título, objetivos, desenho metodológico e considerações finais.

TABELA 1: Distribuição de artigos sobre suicídio de idosos.

\begin{tabular}{|c|c|c|c|}
\hline AUTOR/ ANO & $\begin{array}{l}\text { TÍTULO DO } \\
\text { TRABALHO }\end{array}$ & IDIOMA & BASE DE DADOS \\
\hline $\begin{array}{c}\text { Marques; Sousa; } \\
\text { Nogueira; } \\
\text { Nogueira; Oliveira } \\
(2020)\end{array}$ & $\begin{array}{l}\text { Suicídio em Idosos } \\
\text { Brasileiros: Retrato } \\
\text { de uma realidade }\end{array}$ & Português & Google Acadêmico \\
\hline $\begin{array}{l}\text { Sousa; Mendes; } \\
\text { Carneiro; Oliveira; } \\
\text { Almeida (2019) }\end{array}$ & $\begin{array}{c}\text { O processo de } \\
\text { envelhecimento e } \\
\text { sua relação com o } \\
\text { suicídio na pessoa } \\
\text { idosa: Uma revisão } \\
\text { bibliográfica }\end{array}$ & Português & Google Scholar \\
\hline $\begin{array}{l}\text { Oliveira; Melo; } \\
\text { Aoyama; Lima } \\
\text { (2019) }\end{array}$ & $\begin{array}{l}\text { Suicídio na velhice } \\
\text { como um fenômeno } \\
\text { cada vez mais } \\
\text { crescente e } \\
\text { preocupante }\end{array}$ & Português & Google Scholar \\
\hline $\begin{array}{l}\text { Ferraiuoli; Ferreira } \\
\qquad(2017)\end{array}$ & $\begin{array}{l}\text { O outro lado da } \\
\text { "melhor idade": } \\
\text { Depressão e } \\
\text { suicídio em idosos }\end{array}$ & Português & Google Scholar \\
\hline $\begin{array}{c}\text { Bernardo; Macedo } \\
\text { (2019) }\end{array}$ & $\begin{array}{l}\text { Suicídio no idoso: } \\
\text { Aspectos } \\
\text { Psicossociais que } \\
\text { contribuem para o } \\
\text { Aumento do } \\
\text { Suicídio em pessoas } \\
\text { idosas }\end{array}$ & Português & Google Scholar \\
\hline
\end{tabular}

FONTE: Elaborado pelos autores com dados do Google Scholar.

De forma geral, os resultados apontam para a prevalência de estudos bibliográficos, o que pode indicar a dificuldade de ser feita pesquisa de campo, pois "o 
comportamento suicida ainda é visto como tabu", onde estigmas que envolvem a temática poderá se tornar um obstáculo para pesquisa de campo que aborda a temática sobre suicídio de idosos (BOTEGA, 2015).

A pesquisa de Marques et al. (2020) enfatizou que o suicídio é um inquietante problema de saúde pública e entre idosos é mais planejado e sentenciado do que em outras faixas etárias, o que reforça a necessidade do conhecimento acerca de fatores relacionados com o diagnóstico e o risco do suicídio nessa população, com intuito de identificar, compreender e tratar as vulnerabilidades inerentes a este fenômeno. Pois nessa fase da terceira idade, os idosos com problemas mentais e comportamentais deixam de pedir ajuda, devido à "incapacidade de acessar os serviços de apoio necessários", fato este relevante para uma melhor acessibilidade na existência desses serviços (PAPALIA E FELDMAN, 2013, p.589).

TABELA 2: Distribuição de artigos sobre suicídio de idosos.

\begin{tabular}{|c|c|c|c|}
\hline AUTORES & $\begin{array}{l}\text { OBJETIVO DO } \\
\text { TRABALHO }\end{array}$ & $\begin{array}{c}\text { DESENHO } \\
\text { METODOLÓGICO }\end{array}$ & CONCLUSÃO DA PESQUISA \\
\hline $\begin{array}{c}\text { MARQUES, } \\
\text { Vanessa de Sá } \\
\text { Nobre Formiga; } \\
\text { SOUSA, Milena } \\
\text { Nunes Alves de; } \\
\text { NOGUEIRA, Raquel } \\
\text { Bezerra de Sá de } \\
\text { Sousa; NOGUEIRA, } \\
\text { Tiago Bezerra de } \\
\text { Sá de Sousa; } \\
\text { OLIVEIRA, Isadelia } \\
\text { Constâncio de. }\end{array}$ & $\begin{array}{c}\text { Analisar os fatores } \\
\text { de risco e a } \\
\text { necessidade de } \\
\text { medidas } \\
\text { preventivas } \\
\text { associadas ao } \\
\text { suicídio em idosos } \\
\text { brasileiros }\end{array}$ & $\begin{array}{c}\text { Revisão integrativa } \\
\text { de literatura }\end{array}$ & $\begin{array}{l}\text { Pode-se perceber que } \\
\text { pessoas idosas são um } \\
\text { grupo negligenciado quanto } \\
\text { as políticas públicas de } \\
\text { assistência no processo de } \\
\text { envelhecimento, estando } \\
\text { sujeitos aos mais variados } \\
\text { fatores de risco, entre } \\
\text { outros, que podem resultar } \\
\text { em um maior índice de } \\
\text { suicídio e tentativas } \\
\text { consumadas. A mortalidade } \\
\text { por suicídio em idosos é } \\
\text { maior à medida que a idade } \\
\text { avança, tonando assim, } \\
\text { importante o conhecimento } \\
\text { dos fatores de risco e } \\
\text { intervenção precoce para } \\
\text { melhoria da qualidade de } \\
\text { vida deste grupo. }\end{array}$ \\
\hline $\begin{array}{c}\text { SOUSA, Renata } \\
\text { Maria Assunção de }\end{array}$ & $\begin{array}{l}\text { Analisar estudos } \\
\text { que versam sobre }\end{array}$ & $\begin{array}{c}\text { Revisão } \\
\text { Bibliográfica e }\end{array}$ & $\begin{array}{l}\text { Concluiu-se que o processo } \\
\text { de envelhecimento, é }\end{array}$ \\
\hline
\end{tabular}


PhD Scientific ReviewC ISSN 2676 - 0444

\begin{tabular}{|c|c|c|c|}
\hline $\begin{array}{c}\text { Carvalho; MENDES, } \\
\text { Geovane Soares; } \\
\text { CARNEIRO, } \\
\text { Graziella Freitas da } \\
\text { Costa; OLIVEIRA, } \\
\text { Guilherme Antônio } \\
\text { Lopes de; } \\
\text { ALMEIDA, Márcia } \\
\text { Regina Galvão de. }\end{array}$ & $\begin{array}{l}\text { o processo do } \\
\text { envelhecimento e } \\
\text { sua relação com } \\
\text { suicídio na pessoa } \\
\text { idosa. }\end{array}$ & integrativa & $\begin{array}{l}\text { caracterizado como um } \\
\text { processo dinâmico, } \\
\text { progressivo e irreversível, } \\
\text { ligado intimamente a } \\
\text { fatores biológicos, psíquicos } \\
\text { e sociais. A pessoa idosa é } \\
\text { geralmente a mais atingida } \\
\text { por doenças e por } \\
\text { sentimentos negativos e de } \\
\text { baixa autoestima, a } \\
\text { fragilidade do corpo pode } \\
\text { despertar ou agravar } \\
\text { transtornos psicológicos já } \\
\text { existentes. O tempo livre } \\
\text { ocioso na aposentadoria, } \\
\text { isolamento social, solidão, } \\
\text { distancia ou pouco contato } \\
\text { com a família também } \\
\text { estão entre as motivações } \\
\text { do suicídio em idosos. Com } \\
\text { isso, tem-se como } \\
\text { estratégias de prevenção } \\
\text { campanhas de } \\
\text { sensibilização pública e } \\
\text { cooperação com a mídia } \\
\text { local para melhorar as } \\
\text { atitudes do público em } \\
\text { relação à depressão e } \\
\text { facilitar a busca de ajuda }\end{array}$ \\
\hline $\begin{array}{c}\text { OLIVEIRA, } \\
\text { Francyane Braga } \\
\text { da Silva; MELO, } \\
\text { Kelly Cristina } \\
\text { Ferreira; AOYAMA, } \\
\text { Elisângela de } \\
\text { Andrade; LIMA, } \\
\text { Ronaldo Nunes. }\end{array}$ & $\begin{array}{c}\text { Descrever a } \\
\text { crescente e } \\
\text { alarmante } \\
\text { incidência dos } \\
\text { casos de suicídio } \\
\text { na população idosa } \\
\text { a partir do ano de } \\
2016 .\end{array}$ & $\begin{array}{c}\text { Pesquisa } \\
\text { bibliográfica por } \\
\text { meio de uma } \\
\text { revisão integrativa } \\
\text { (RI) de literatura. }\end{array}$ & $\begin{array}{l}\text { Os números sobre o suicídio } \\
\text { entre idosos mostram } \\
\text { incidência crescente e } \\
\text { alarmante; Dados ainda } \\
\text { insuficientes para a } \\
\text { compreensão deste } \\
\text { fenômeno em sua } \\
\text { totalidade, eles alertam } \\
\text { para a necessidade de se } \\
\text { dar mais atenção ao risco } \\
\text { de suicídio entre idosos e } \\
\text { novos estudos ainda são } \\
\text { necessários sobre esse } \\
\text { tema; no entanto, concluiu- } \\
\text { se que o suicídio nos idosos }\end{array}$ \\
\hline
\end{tabular}




\begin{tabular}{|c|c|c|c|}
\hline & & & $\begin{array}{c}\text { está diretamente } \\
\text { relacionado a fatores } \\
\text { psicológicos a maioria } \\
\text { possuía diagnósticos } \\
\text { anterior a morte, tendo o } \\
\text { transtorno depressivo a } \\
\text { maior prevalência. As } \\
\text { relações familiares parecem } \\
\text { ter influenciado na } \\
\text { qualidade de vida dos } \\
\text { idosos. }\end{array}$ \\
\hline $\begin{array}{c}\text { FERRAIUOLI, } \\
\text { Ceneida; } \\
\text { FERREIRA, Scheilla } \\
\text { Maria Ribeiro } \\
\text { Rocha. }\end{array}$ & $\begin{array}{l}\text { Discutir sobre o } \\
\text { transtorno } \\
\text { depressivo entre } \\
\text { os idosos, bem } \\
\text { como analisar os } \\
\text { fatores } \\
\text { preponderantes } \\
\text { que levam um } \\
\text { indivíduo nesta } \\
\text { faixa etária a } \\
\text { desistir da vida. }\end{array}$ & $\begin{array}{l}\text { Revisão de } \\
\text { literatura. }\end{array}$ & $\begin{array}{l}\text { Foi possível concluir que } \\
\text { além da depressão, as } \\
\text { enfermidades físicas, o } \\
\text { isolamento social e os } \\
\text { fatores psicossociais } \\
\text { também possuem grande } \\
\text { influência na decisão dos } \\
\text { idosos. }\end{array}$ \\
\hline $\begin{array}{l}\text { BERNARDO, } \\
\text { Beatriz Taconi; } \\
\text { MACEDO, } \\
\text { Fernando Luis. }\end{array}$ & $\begin{array}{c}\text { Identificar e } \\
\text { descrever } \\
\text { características } \\
\text { importantes que } \\
\text { contribuem para o } \\
\text { suicídio no idoso. }\end{array}$ & $\begin{array}{l}\text { Revisão de } \\
\text { literatura. }\end{array}$ & $\begin{array}{l}\text { Concluiu-se o suicídio no } \\
\text { idoso como vindo de } \\
\text { fatores multifatoriais, } \\
\text { depressão, gravidades } \\
\text { físicas, mentais, fatores } \\
\text { sociais, perda no padrão de } \\
\text { vida também potencializam } \\
\text { a vontade de tirar a própria } \\
\text { vida. As questões } \\
\text { existenciais como } \\
\text { desesperança, ou não } \\
\text { encontrar sentido na vida } \\
\text { podem potencializar o } \\
\text { desejo de morrer. }\end{array}$ \\
\hline
\end{tabular}

FONTE: Elaborado pelos autores com dados do Google Scholar.

Sendo considerado um problema de saúde pública, o suicídio de idosos tem suas demandas específicas que devem ser voltadas, segundo Melo (2018) apud Marques et al (2020) como sendo: 
[...] compreensão nas necessidades de saúde, assim como ações multidisciplinares voltadas à população idosa, com foco na prevenção da capacidade funcional e cognitiva continua sendo um enorme desafio para o Sistema Único de Saúde, já que diversos profissionais devem colaborar conjuntamente para tornar possível uma abordagem integrativa" (MARQUES et al, 2020).

O enfrentamento deste grave problema se faz necessário, pois o suicídio de acordo com alguns estudos está associado a problemas mentais, como a depressão e também a falta de interação social e assim a necessidade de tais ações multidisciplinares.

O suicídio também é visto por Sousa; Mendes; Carneiro; Almeida (2019) como um problema de saúde pública, especialmente em idosos, pois esse grupo está propenso a diversas doenças, inclusive a depressão, que geralmente está vinculada ao estado de grande sofrimento físico; fato este reforçado por Teixeira e Martins (2018), ao afirmar que, "contudo, alguns fatores suicidógenos apontados pela literatura devem ser levados a sério, especialmente por potencializarem significativamente o risco de suicídio quando aparecem vinculados a circunstâncias intensificadoras do sofrimento psíquico de idosos" (TEIXEIRA E MARTINS, 2018, P. 263). Considera-se este um grande fator de risco, pois “a pessoa idosa é geralmente a mais atingida por essas doenças e por esses sentimentos negativos e de baixa autoestima, a fragilidade do corpo pode despertar ou agravar transtornos psicológicos já existentes" (CAVALCANTE; MINADO; MANGAS, 2013 APUD SOUSA; MENDES; CARNEIRO; ALMEIDA, 2019), e assim agravar o estado do idoso, sabendo-se que são permeados de situações vivenciadas nessa fase como os mencionados por Sousa et al (2019) que é "O tempo livre ocioso na aposentadoria, isolamento social, solidão, distancia ou pouco contato com a família também estão entre as motivações do suicídio em idosos; assim, fatores mencionados anteriormente são fortemente desencadeadores do suicídio em idosos.

Já Oliveira et al (2019) enfatizam as medidas preventivas em relação ao suicídio, pois mesmo considerando o alerta de saúde pública, diz que alguns fatores contribuem para o enfrentamento das situações as quais são adversas para a vida dos idosos e colocando-os como essenciais na prevenção ao suicídio, como: 
Um bom vínculo com a família é um fator importantíssimo e que previne de forma clara o suicídio. O sentimento de solidão ou de abandono pode evidenciar as ideações suicidas. Outro fator é o apoio da sociedade aos idosos, especialmente os que sofreram algum tipo de violência doméstica. A atividade religiosa também tem grande papel na prevenção do suicídio na população idosa e por fim o acesso a serviços de saúde tem um peso grandioso, pois o idoso requer atenção individualizada e terapia medicamentosa ou não medicamentosa no enfrentamento à ideação suicida e ao suicídio, evitando assim que ele tenha uma visão negativa a cerca da própria vida (OLIVEIRA; MELO; AOYAMA; LIMA, 2019, p. 4).

Oliveira et al $(2019$, p.4) ressaltam em sua pesquisa que "os transtornos mentais e as doenças psicológicas possuem relação estreita com o suicídio de idosos, especialmente a depressão", enfatizando, assim, a depressão como ocupando um papel de impacto principalmente quando associada a outros determinantes.

Em sua pesquisa, Ferraiouli e Ferreira (2017) abordaram sobre os métodos utilizados no suicídio cometido por idosos, como o enforcamento, o envenenamento e o uso de arma de fogo, ressaltando que "os principais fatores que levam o idoso ao ato suicida são a depressão, as enfermidades físicas, o isolamento social e os fatores psicossociais" (FERRAIUOLI; FERREIRA, 2017, p. 50), então se torna importante o conhecimento, os fatores e os métodos utilizados no acometimento do ato suicida de idosos.

A pesquisa de Corrêa, 2018 apud Bernardo e Macedo (2019, p.64) faz orientações importantes às pessoas que tem acesso aos idosos, pois "orienta aos familiares e amigos que fiquem atentos a alguns sinais que sinalizam o adoecimento mental, como a depressão". Segundo Bernardo e Macedo (2019), ao analisar os aspectos psicossociais do suicídio em pessoas idosas, destaca o que diz Assunção; Oliveira e Sousa, 2018.

\footnotetext{
A depressão é um problema mundial podendo afetar desde adolescência até a velhice, causando muita tristeza, angústia e perda considerada de interesse pela vida. Foi verificada uma correlação entre suicídio e depressão, e altos números de pessoas depressivas efetivarem a retirada da própria vida; [...], estudos mostraram uma relação entre suicídio e o idoso aos transtornos mentais e principalmente, associados à depressão maior (BERNARDO; MACEDO, 2019 apud ASSUNÇÃO; OLIVEIRA, 2018).
}

Então é preciso levar em consideração, além de tudo o que foi analisado, o papel das pessoas que estão responsáveis pelos cuidados dos idosos e as orientações 
quanto os desencadeadores do suicídio, quanto sobre a crise suicida que segundo Botega (2013) ocorre da seguinte forma:

[...] na crise suicida, há a exacerbação de uma doença mental existente, ou uma turbulência emocional que, sucedendo um acontecimento doloroso, é vivenciada como um colapso existencial. Ambas as situações provocam dor psíquica intolerável e, como consequência, pode surgir o desejo de interrompê-la por meio de cessação do viver (BOTEGA,2013, P. 13).

Então, com essas orientações, o reconhecimento de uma crise suicida, a importância do cuidador que além do seu papel de acolher, auxiliar o idoso nos seus cuidados pessoais e assim desenvolver certa autonomia gerando um ambiente de segurança para o idoso, vai ter o papel fundamental de alertar a família ao reconhecer um comportamento suicida no idoso e assim servindo como meio de prevenção ao suicídio do idoso ao qual está sendo cuidado e tendo uma melhor qualidade de vida.

Portanto, os cinco artigos apresentaram uma base de desenho metodológico coerente às informações em torno dos resultados que mostram a depressão associada a outros fatores como um transtorno mental que necessita ser prevenido e cuidado na fase do envelhecimento, ressaltando esses resultados como fidedignos ao de Teixeira e Martins (2018), onde, concluíram que:

[...] mediante a construção de autópsias psicossociais, tornou-se possível identificar os possíveis fatores psicossociais associados ao suicídio desses idosos: vida desprovida de sentidos, depressão, relações afetivas fragilizadas, comportamentos autodestrutivos como uso abusivo de álcool, traços de personalidade impulsivo-agressiva e ocorrência de tentativas de suicídio anteriores (TEIXEIRA E MARTINS, 2018; p.268).

Desse modo, segundo as pesquisas analisadas a depressão é fator de risco para o suicídio de idosos, mas Botega (2013), faz uma ressalva diante de que:

Ainda que uma doença mental esteja presente na maioria dos casos, isso não é obrigatório. O comportamento suicida em geral indica um sofrimento profundo, mas não necessariamente uma doença mental. É importante lembrar, também, que a maioria das pessoas acometidas por transtorno mental não se mata (BOTEGA E MARTINS,2013, p.36).

Porém, a terceira idade, por ser uma fase de vulnerabilidades, Papalia e Feldman (2013, p.649) apontam a depressão como fator de risco ao suicídio, pois "geralmente apresentam sinais de depressão, como uma incomum dificuldade para se concentrar, perda de autoestima e sentimentos de desamparo, desesperança ou 
pânico"; logo, a depressão associada a outros fatores de riscos ao suicídio, se apresenta permeando tal contexto que é o fenômeno do suicídio entre idosos.

\section{CONSIDERAÇÕES FINAIS}

Concluímos que a depressão associada a outros fatores como o sofrimento físico, questões existenciais, isolamento social, entre outros, potencializam a consumação do suicídio em idosos, onde a ênfase na depressão contribui para identificação dos fatores de riscos associados ao suicídio em idosos e uma melhor análise de caráter preventivo ao passo que todos os artigos mostraram a confirmação do suicídio de idosos como necessidade de saúde pública e que a depressão por ter sido vista com prevalência associada ao suicídio de idosos, requer uma atenção maior aos cuidados com a saúde mental.

Sugere-se que as novas pesquisas sejam feitas ampliando as bases de pesquisas e que sejam realizadas a partir de estudos de campo feitos no Brasil como em outros países para serem desenvolvidos e difundidos mais conhecimentos em torno do fenômeno do suicídio e possíveis prevenções contra esse fenômeno ainda permeado de tabus, de estigmas em torno dos comportamentos suicidas e obstáculos para a busca de orientações. 


\section{REFERÊNCIAS}

BOTEGA, Neury José. Crise suicida: avaliação e manejo [recurso eletrônico] / Neury José Botega. - Porto Alegre : Artmed, 2015. e-PUB Desenvolvimento Humano [recurso eletrônico] / Diane E. Papalia, Ruth Duskin Feldman, com Gabriela Martorell ; tradução : Carla Filomena Marques Pinto Vercesi... [et al.] ; [revisão técnica: Maria Cecília de Vilhena Moraes Silva... et al.]. - 12. ed. - Dados eletrônicos. - Porto Alegre : AMGH, 2013.

DE OLIVEIRA TEIXEIRA, Selena Mesquita; DE OLIVEIRA MARTINS, José Clerton. 0 suicídio de idosos em teresina: fragmentos de autópsias psicossociais. Fractal: Revista de Psicologia, p. 262-270, 2018.

DE CARVALHO SOUSA, Renata Maria Assunção et al. O processo de envelhecimento e sua relação com o suicídio na pessoa idosa: uma revisão bibliográfica. In: Congresso Brasileiro de Assistentes Sociais 2019.

DA SILVA OLIVEIRA, F. B.; MELO, K. C. F., LIMA, R. N.; de ANDRADE AOYAMA, E. (2019). Suicídio na velhice como um fenômeno cada vez mais crescente $e$ preocupante. Revista Brasileira Interdisciplinar de Saúde.

FERRAIUOLI, Ceneida; FERREIRA, Scheilla. O outro lado da" melhor idade": depressão e suicídio em idosos. Perspectivas Online: Humanas \& Sociais Aplicadas, v. 7, n. 18, 2017.

FUMEGALLI, Ana. Taxa de suicídio entre idosos cresce e prevenção é o melhor caminho. 20 maio 2021. Disponível em: https://saude.rs.gov.br/taxa-de-suicidio-entreidosos-cresce-e-prevencao-e-o-melhor caminho\#.X9OVRyQwVz0.whatsapp. Acesso em: 17 maio 2021.

GIL, Antônio Carlos. Métodos e técnicas de pesquisa social- 6. ed. - São Paulo : Atlas, 2008.

MARQUES, Vanessa de Sá Nobre Formiga et al. suicídio em idosos brasileiros: retrato de uma realidade. Revista Brasileira Multidisciplinar, v. 23, n. 3, p. 190-202, 2020.

MACEDO, Fernando Luis; BERNARDO, Beatriz Taconi. Suicídio no idoso: aspectos psicossociais que contribuem para o aumento do suicídio em pessoas idosas. Revista InterCiência-IMES Catanduva, v. 1, n. 3, p. 60-60, 2019.

SANTOS, José; $\quad$ ENCARNAÇÃO, Maria Fernanda Neves da Silva. Modernidade e gestão da velhice. Centro Regional de Segurança Social do Algarve, 1998. 336 p. 